den, dass das Freiheitsrecht eines Elternteils nur eingeschränkt werden kann, wenn das Kindeswohl dies zwingend erfordert. Andere Entscheidungen forderten beachtenswerte, insbesondere berufliche Gründe, die der ausreisewillige Elternteil darlegen musste. ${ }^{12}$ Das OLG Köln sah die Freizügigkeit pflichtgebunden in Hinblick auf das Kindeswohl, es müssten triftige Gründe für den Wegzug vorliegen. ${ }^{13}$ Nur dann sei es auch gerechtfertigt, das Elternrecht des anderen und sein Umgangsrecht einzuschränken.

Der BGH hat nun diese Fragen eindeutig beantwortet. Mit seinem Beschluss vom 28. April $2010^{14}$ hat er klargestellt, dass das Kindeswohl „vornehmlich“ Maßstab der Entscheidung sei. Die allgemeine Handlungsfreiheit des auswanderungswilligen Elternteils schließe es aus, die Möglichkeit seines Verbleibes im Inland als Alternative in Betracht zu ziehen. Die Gründe und Motive für seinen Auswanderungswunsch seien nur insoweit bedeutsam, als sie sich auf das Kindeswohl auswirken. Das wiederum sei aber gegeben, wenn etwa mit der Auswanderung (vorwiegend oder beiläufig) auch der Zweck verfolgt wird, den Kontakt des Kindes mit dem anderen Elternteil zu vereiteln, denn dann ,steht die Bindungstoleranz des betreuenden Elternteils und somit seine Erziehungseignung in Frage“.

Die Kindeswohl ist umfassend zu prüfen: Der gesamte Senat, nicht nur eine Berichterstatterin oder ein Berichterstatter, hat das Kind anzuhören ${ }^{15}$, eine Verfahrenspflegerin oder ein Verfahrenspfleger ist zu bestellen und bei der Anhörung hinzuzuziehen. ${ }^{16}$ Die Entscheidung fällt dann danach, „ob die Auswanderung mit dem Elternteil oder der Verbleib des Kindes beim weiter im Inland ansässigen Elternteil die für das Kindeswohl bessere Lösung ist ${ }^{17}$. Hierbei sind die Bindungen des Kindes zu klären: Überwiegt diejenige an die auswandernde Hauptbezugsperson oder sind es andere Bindungen, zum Beispiel an die gewohnte Umgebung und das gesamte, ver- traute Umfeld, ferner der vom Kind geäußerte Wille (welcher bei kleinen Kindern mehr als Ausdruck ihrer Bindungen und bei älteren eher als Ausdruck ihrer Persönlichkeit zu werten ist). Falls erforderlich, ist ein Sachverständigengutachten einzuholen. Auch ist die Alternative eines Wechsels zum bleibenden Elternteil in Betracht zu ziehen.

Fälle, die brisant sind, wie der eingangs geschilderte, sind zum Glück nicht die Regel. Aber Ehen zwischen deutschen und ausländischen Partnerinnen und Partnern, die in den 50er Jahren des letzten Jahrhunderts noch etwas Exotisches waren, sind heute etwas völlig Normales und recht zahlreich: Im Jahre 2008 waren 10,9 Prozent aller in Deutschland geschlossenen Ehen (41.442 von 377.055) binational, und diese Quote ist konstant. ${ }^{18}$ Von allen in Deutschland geborenen Kindern entstammen zwischen 12 und 13 Prozent binationalen Ehen, das waren beispielsweise im Jahre 200686.744 Kinder. ${ }^{19} \mathrm{Ihr}$ Wohlergehen ist nach den neuen BGH-Entscheidungen das vorrangige und entscheidende Kriterium dafür, ob sie ihren Aufenthalt in Deutschland beenden und mit ihrem ausländischen Elternteil wegziehen (müssen oder dürfen).

12 OLG München, FamRZ 2008, S. 1774, OLG Nürnberg, FamRZ 2010, S. 135.

13 FamRZ 2009, S. 436.

14 FamRZ 2010, S. $1060 \mathrm{ff}$.

15 Dies wurde in einer neueren Entscheidung bestätigt, dass generell der gesamte Senat anhören und dabei die Verfahrenspflegerin oder der Verfahrenspfleger anwesend sein muss, vgl. BGH, FamRZ 2011, S. 800.

16 Wobei sich diese eingehend mit dem Fall befasst haben muss und nicht erst kurz vor dem Termin nur 30 Minuten mit dem Kind gesprochen und die Akten "selektiv“ gelesen haben darf; KG, FamRZ 2011, S. 800.

17 BGH, FamRZ 2010, S. 1063.

18 Auskunft des Bundesministeriums für Familie, Senioren, Frauen und Jugend vom 8.6.2011 an die Autorin.

19 S. iaf, a.a.O, vorletzte Seite.

\title{
Erbrecht im Visier: Wann kommt das Erbrecht im 21. Jahrhundert an?
}

\section{Renate Maltry}

Vizepräsidentin des djb; Rechtsanwältin, Fachanwältin für Erbrecht, Fachanwältin für Familienrecht, München

Im Vergleich zum Familienrecht, das sich fortlaufend dem gesellschaftlichen Wandel angepasst hat, erstaunt, dass das Erbrecht bisher kaum Veränderung bzw. Anpassung an geänderte gesellschaftliche Strukturen erfahren hat.

Das Erbrecht ist der Teil des BGB, der seit seinem Inkrafttreten im Jahre 1900 mit wenigen Ausnahmen noch unverändert geblieben ist.

Es ist an der Zeit über den Einfluss des familiären und demografischen Wandels im Erbrecht nachzudenken und Refor- men, die einer modernen Gesellschaft gerecht werden, einzufordern.

\section{Demografischer Wandel}

Betrug das durchschnittliche Lebensalter im Jahre 1881 bei Frauen 38,45 Jahre und bei Männern 35,6 Jahre, so hat sich die Lebenserwartung bei Männern um 40 Jahre und bei Frauen um 43 Jahre erhöht. ${ }^{1}$ Folge hiervon ist, dass die sogenannten jungen Alten, also die ca. 60-Jährigen, von den sehr Alten erben.

1 Statistisches Bundesamt: Statistisches Jahrbuch 2007. 


\section{Familiärer Wandel}

Wurden im Jahre 1900 von 476.491 Eheschließungen 9.152 Ehen geschieden, stieg der Anteil im Jahre 1955 auf 74.018 Scheidungen bei 617.228 Eheschließungen und erreichte seinen bisherigen Höhepunkt im Jahre 2003 mit 213.975 Scheidungen auf 382.911 Eheschließungen. Betroffen waren davon 143.437 Kinder. $^{2}$

Folge ist die Polarisierung von Familienformen. Besonders auffällig ist die Zunahme von Stief- und Patchworkfamilien, Kinderlosigkeit und unverheirateten Lebenspartnerschaften.

Die Rolle der Frau hat sich nicht zuletzt durch die Stärkung des Grundsatzes der Eigenverantwortung im Unterhaltsrecht und ihre Verpflichtung zur Erwerbstätigkeit gänzlich gewandelt. Rollenmuster in einer Ehe und Partnerschaft sind heute vielfältig, asymmetrisch, arbeitsteilig und immer noch selten gleichgestellt.

Welche Bedeutung hat dies für einen Veränderungsbedarf im Erbrecht?

\section{Gesetzliches Erbrecht}

Nach wie vor unterliegen die meisten Nachlassfälle der gesetzlichen Erbfolge.

Das bestehende gesetzliche Erbrecht geht von der Anknüpfung des Erbrechts an den Güterstand aus.

Dies ist gemäß $\mathbb{1 9 3 1}$ I BGB davon abhängig, ob Abkömmlinge oder Eltern und Großeltern vorhanden sind. Neben den Verwandten der zweiten Ordnung, also Eltern und Großeltern, erhöht sich der Erbteil des Ehegatten auf die Hälfte. Neben Abkömmlingen beträgt der gesetzliche Erbteil ein Viertel.

Nur wenn die Eheleute in Zugewinngemeinschaft gelebt haben, erhöht sich der Anteil des gesetzlichen Erbteils um die pauschale Zugewinnquote neben Verwandten der ersten Ordnung; also neben Kindern auf die Hälfte und neben Verwandten der zweiten Ordnung auf drei Viertel gemäß $\mathbb{S} 1931$ III, 1371 BGB (erbrechtliche Lösung), sofern nicht der Zugewinn auszugleichen ist und das Wahlrecht der Ausschlagung mit Geltendmachung des Pflichtteils ausgeübt wird, $\mathbb{S} 1371$ III BGB (güterrechtliche Lösung).

Beim Güterstand der Gütergemeinschaft, $\mathbb{S} 1415$ BGB, kommt es nicht zur Erhöhung nach den oben genannten güterrechtlichen Bestimmungen, so dass es bei der Erbquote des überlebenden Ehegatten, also einem Viertel neben Verwandten der ersten Ordnung und drei Vierteln neben Eltern und Großeltern, verbleibt.

Gleiches gilt beim Güterstand der Gütertrennung nach \1414 BGB. Zusätzlich ist hier jedoch die Erbquote abhängig von der Anzahl der Kinder, $\mathbb{S} 1931$ IV BGB, und beträgt bei einem Kind die Hälfte und bei zwei Kindern ein Drittel. Die Begrenzung erfährt die Erbquote bei einem Viertel, $\mathbb{1 9 3 1}$ BGB.

Die Mehrheit der Bevölkerung hat von dem Zusammenhang zwischen Erbrecht und Güterstand keine Kenntnis, sondern geht davon aus, dass die überlebende Ehepartnerin oder der überlebende Ehepartner nach dem Tod der oder des anderen immer zur Hälfte berufen sei. ${ }^{3}$
Nach einer Studie des BMFSFJ, der Sinus Sociovision GmbH, Heidelberg, vom Januar 2011 kennen die meisten Frauen und Männer den rechtlichen Rahmen und die Koordinaten der Institution Ehe nicht. Elemente wie Gütertrennung und Zugewinngemeinschaft und deren Folgen sind ihnen fremd. ${ }^{4}$ Hintergründe sind das Grundvertrauen in die Zuständigkeit und Verantwortung des Staates gemäß der Alltagsphilosophie der „Vollkasko-Delegation“, so die vorgenannte Studie.

Dies kann nur ein Handlungsauftrag an den Gesetzgeber sein, der verlässliche Rahmenbedingungen schaffen muss und eine Vereinheitlichung und Vereinfachung der gesetzlichen Erbfolge vorzunehmen hat.

Diskutiert wird seit einigen Jahren in Deutschland wie auch in den uns umgebenden Rechtsordnungen die Frage nach einer Verstärkung des Ehegattenerbrechts bzw. des Erbrechts eingetragener Lebenspartnerschaften (sog. Horizontalisierungstendenz)..$^{5}$

Der 68. Deutsche Juristentag (djt) 2010 hat sich dafür ausgesprochen, das Erbrecht des überlebenden Ehegatten güterstandsunabhängig auf die Hälfte neben einem und mehreren Kindern und auf drei Viertel neben übrigen Verwandten festzusetzen. $^{6}$

Der Preis hierfür soll sein, dass neben der Erhöhung der Erbquote ein realer Zugewinnausgleich nicht stattfinden soll. ${ }^{7}$

$\mathrm{Ob}$ eine weitere Verstärkung des Ehegattenerbrechts bis hin zur Alleinerbschaft eintreten soll, ist wohl eine Frage der gesellschaftlichen Wertung und des Schutzes von langjährigen Ehen.

Bei langjährigen Ehen sind die Kinder in der Regel durch die während der Ehe vorgenommenen Zuwendungen weniger auf den erbrechtlichen Erwerb angewiesen.

In Erwägung gezogen werden sollte auch die Frage nach einem Wohnrecht der verbleibenden Ehepartnerin oder des verbleibenden Ehepartners, die oder der bei fehlendem Testament oder Geltendmachung von Ansprüchen der Kinder ihr oder sein Zuhause verlassen muss, um eventuelle Ausgleichszahlungen leisten zu können. Die verbesserte Stundungsregelung schafft hier schon wegen des Zinsanspruches und einer meist angeordneten Sicherheitsleistung keine Abhilfe.

Aus den Erfahrungen, die bei den Neuregelungen zum Familienrecht gemacht wurden, sollte dem Schutz der langjährigen Ehen besonderes Augenmerk geschenkt werden. Letztendlich unterliegt die Ehe dem Schutz des Artikels 6 GG, wobei die Eheleute in der Wahl und Aneinanderreihung des Ehetypus grundsätzlich frei sind.

Nimmt man jedoch auf der anderen Seite die Scheidungsstatistiken und die Zahl der Zweit- und Drittehen ernst, so relativieren sich Begriff und Bedeutung der Ehebeziehung.

\footnotetext{
2 Statistisches Bundesamt: Statistisches Jahrbuch 2007.

3 Vgl. Littig, Thomas, FPR 2011, S. 241.

4 S. Studie des BMFSFJ der Sinus Sociovision $\mathrm{GmbH}$, Heidelberg, Januar 2011, S. 62/63.

5 Vgl. Röthel, Reformbedarf im Erbrecht - aus Sicht der Wissenschaft, ERbR 06/2011, S. 167.

6 Beschluss Nr. 17 des 68. DJT 2010, Streichung des $\S 1331$ Abs. 3 BGB.

7 Beschluss Nr. 18 des 68. DJT 2010.
} 
Diese wird immer flüchtiger. Auch besteht die Gefahr, dass der Trend zur jungen Ehefrau oder - seltener - zum jungen Ehemann in der letzten Lebensphase durch die Verstärkung des Ehegattenerbrechts begünstigt wird.

Eine endgültige Lösung kann dieser Ansatz daher nicht sein. Die Kommission Zivil-, Familien- und Erbrecht, Recht anderer Lebensgemeinschaften des djb wird sich mit diesem Thema auseinandersetzen. Möglicherweise werden auch ganz neue und andere Ansatzpunkte entwickelt.

\section{Gewillkürte Erbfolge}

Viele Kautelarjuristinnen und -juristen sehen angesichts der Flexibilität und der Gestaltungsmöglichkeiten der Kautelarpraxis keinen Reformbedarf im Erbrecht. ${ }^{8}$

Folgt man dieser Ansicht, müsste die Anzahl der Testamente proportional zum Anstieg der Scheidungsrate sein. Die Anzahl der Testamente pro Bundesbürger liegt jedoch nur bei vier Prozent.

Zudem sind bereits vorhandene Möglichkeiten wie etwa die Jastrowsche Klausel, das Geschiedenen-Testament, Vorund Nacherbklauseln, Berliner Testament etc. meist nicht ausreichend, den Willen der Erblasserin oder des Erblassers umzusetzen. Die Testiermöglichkeiten sind erheblich eingeschränkt durch Pflichtteils- und Erbschaftssteuerlasten. Insbesondere in Patchworkfamilien reichen die bestehenden Erbrechtsinstrumente nicht aus, eine zufriedenstellende Gestaltung zu ermöglichen.

Es sollte folglich ein gesetzliches Leitbild für die Rechtsfolgen der Wiederverheiratung und die Nachlassaufteilung zwischen Erst- und Zweitfamilie eingefügt werden.

Änderungsbedarf besteht auch im Hinblick auf das gemeinsame Testament an sich, $\mathbb{S} \mathbb{S} 2265$ ff. BGB; $\mathbb{S} 10$ LPartG. Häufig scheint das Testament - ob unter Druck oder nicht nur gegengezeichnet zu sein.

Die Folge beispielsweise von wechselbezüglichen Verfügungen oder der mangelnden Möglichkeit der Quotenänderung bei Kindern als Schlusserben ist vielen nicht bewusst. Vielfach wird die Bindung durch wechselbezügliche Verfügungen nicht erkannt und erst nach dem Todesfall festgestellt, dass keine Handlungsfähigkeit mehr besteht.

Bei der Errichtung des gemeinsamen Testaments ohne Rechtsberatung kommen Fehlvorstellungen über Bedeutung und Rechtsfolgen der Errichtung häufig vor.

Vergleicht man die in der Reform des Unterhaltsrechts eingeführte Voraussetzung der gerichtlichen Protokollierung bzw. notariellen Beurkundung von Unterhaltsvereinbarungen, $\mathbb{} \int 1585$ c BGB, so ist der mangelnde Schutz bei so tragenden Regelungen im Erbrecht nicht nachzuvollziehen. Mindestvoraussetzung für die Wirksamkeit des gemeinsamen Testamentes sollte eine anwaltliche Beratung sein.

\section{Vorweggenommene Erbfolge}

Mit zunehmender Lebenserwartung werden Vorempfänge und Übertragungen zu Lebzeiten auf Kinder häufiger. Die Regelung, dass die Anrechnung nur bei Anordnung der Erblasserin oder des Erblassers bei der Zuwendung erfolgt, $\mathbb{} 2050$ III BGB, ist seit langem in der Kritik und führt zu ungerechten Ergebnissen in der Verteilung zwischen den Abkömmlingen. Vielen ist die Regelung des $\$ 2050$ III BGB weder bekannt noch bewusst. Sinnvoller wäre festzulegen, dass die Zuwendung dann nicht angerechnet wird, wenn dies ausdrücklich festgehalten ist.

\section{Schutz vor einseitiger Interessendurchsetzung}

Proportional zum Anstieg der Lebenserwartung steigt auch die Demenzhäufigkeit, so dass bei den 90-Jährigen jede/r zweite an Demenz erkrankt ist. ${ }^{9}$

Die Übergänge zur Testierunfähigkeit sind schleichend. Im Prozess ist der Beweis der Testierunfähigkeit zu führen, der meist schwer gelingt.

Die Erblasserin oder der Erblasser ist gegen die mit steigendem Lebensalter verbundene Gefährdung vor unbedachter und ungewollter Testierung zu schützen. Die Fremdbestimmungen sollten genau identifiziert und wirkungsvoll sanktioniert werden.

Das Verbot der Erbeinsetzung hinsichtlich Pflegepersonen, $\$ 14$ HeimG, sollte auf Betreuerinnen und Betreuer ausgeweitet werden.

\section{Wegfall des gesetzlichen Erbrechts}

Das gesetzliche Erbrecht der Ehepartner bzw. eingetragenen Lebenspartner sollte dann entfallen, wenn die Auflösung der Solidarverbindung folgt.

\ 1933 BGB, der den Erbeintritt von der Zustellung des Scheidungsantrags bzw. der Zustimmung hierzu abhängig macht, ist seit langem in der Diskussion und nicht mehr akzeptabel.

Sinnvoll wäre, dass die Erbberechtigung mit Wirkung des Gestaltungsurteils, d.h. mit Rechtskraft des Urteils entfällt. Dies würde Klarheit schaffen.

\section{Pflegeleistungen}

Geregelt sind gemäß $\$ 2057$ a BGB nach wie vor nicht die Pflegeleistungen von Schwiegerkindern oder Lebensgefährtinnen und Lebensgefährten, die der djb schon mit der letzten Reform eingefordert hat. Werden sie zu Lebzeiten nicht entlohnt oder in der Testamentsgestaltung nicht bedacht, gehen sie hinsichtlich der erbrachten Leistungen leer aus. Im Fall der Erbringung der Leistung zum Beispiel durch die Schwiegertochter kann der Ehemann zwar die Leistung für sich beanspruchen, die Schwiegertochter jedoch nicht. Dies ist mit ehelicher Solidarität nicht zu vereinbaren und widerspricht dem im Unterhaltsrecht aufgestellten Grundgedanken der Eigenverantwortung.

\section{Verfahrensrecht}

Last but not least sollte an eine Vereinfachung des Verfahrensrechts wie im Familienrecht durch die Schaffung des großen Familiengerichtes gedacht werden. Es ist den Erbenden schwer

8 So Mayer, Gewillkürte Erbfolge, FUR 6/2011, S. 251.

9 Statistisches Bundesamt: Statistisches Jahrbuch 2007. 
zu erklären, warum sie ein Erbscheinsverfahren benötigen und im Anschluss hieran noch bei einem anderen Gericht die Erbenfeststellung vornehmen müssen. So wie das große Familiengericht eingeführt wurde, ist sicherlich auch im Erbrecht Klarheit und Vereinfachung durch ein großes Nachlassgericht herbeizuführen.

\section{Fazit}

Auch der letzte djt 2010 in Berlin hat das Thema unter dem Motto „Ist unser Erbrecht noch zeitgemäß?“ aufgegriffen.
Die djb-Kommission wird sich dem Thema stellen und die entsprechenden frauenpolitischen Zusammenhänge herausarbeiten.

Anpassungen müssen auch schon aufgrund der Entwicklungen auf europäischer Ebene erfolgen.

Noch gehören die jetzt Versterbenden nicht der Scheidungsgeneration an. Es ist jedoch an der Zeit, weiter in die Diskussion einzutreten und das Erbrecht vorausschauend den Anforderungen der sich geänderten und noch ändernden Gesellschaft anzupassen.

\section{Die Reform des Unterhaltsrechts - eine unendliche Geschichte für die (geschiedene) Ehefrau?}

\section{Brigitte Meyer-Wehage \\ Mitglied der Kommission Zivil-, Familien- und Erbrecht, Recht an- derer Lebensgemeinschaften des djb; Richterin am AG, Oldenburg}

Das Unterhaltsrechtsänderungsgesetz vom 21. Dezember 2007, welches zum 1. Januar 2008 in Kraft getreten ist, führt mit seinen Neuerungen - bezogen auf den nachehelichen Unterhalt - in der Praxis zu „echten Streitfällen“. 1

Ein kurzer Rückblick: Die Diskussion um die Reform des Unterhaltsrechts wurde durch die Entscheidung des Bundesverfassungsgerichts vom 28. Februar $2007^{2}$ in eine andere Richtung gelenkt. Denn das höchste deutsche Gericht hatte dem Gesetzgeber in die Agenda geschrieben, dass es gegen $\mathrm{Ar}-$ tikel 6 Absatz 5 GG verstoße, die Dauer des Unterhaltsanspruchs eines Kindes daran festzumachen, ob es ehelich oder nichtehelich geboren ist. Zur Erinnerung: Der Unterhalt gemäß \ 1570 BGB war vor der Reform von dem sogenannten „Altersphasenmodell“ geprägt, während $\mathbb{} 16151$ Absatz 2 Satz 3 BGB alte Fassung der Mutter Unterhalt grundsätzlich nur bis zum vollendeten dritten Lebensjahr des Kindes zubilligte.

Der Gesetzgeber hat - in Umsetzung der Entscheidung - alsdann einen auf drei Jahre befristeten „Basisunterhalt“ eingeführt, der aus Gründen der Billigkeit verlängert werden kann. ${ }^{3}$

Dies war der erste tiefe Einschnitt in das bisherige „System" gefolgt von dem nun kodifizierten Grundsatz der Eigenverantwortung. ${ }^{4}$

\section{Entwicklung der Rechtsprechung}

\section{Betreuungsunterhalt}

Mit Urteil vom 16. Juli $2008^{5}$ äußerte sich der Bundesgerichtshof (erstmals) zur „Bemessung“ der Ansprüche gemäß \S 1615l, 1570 BGB nach „Höhe und Dauer“.

Danach trifft die Kindesmutter ab dem vollendeten dritten Lebensjahr des (jüngsten) Kindes grundsätzlich eine Erwerbspflicht. Weitergehende Ansprüche sind im Einzelfall bei „kindund elternbezogenen“ Gründen unter Billigkeitserwägungen zu prüfen, wobei die Gründe von der oder dem Unterhaltsberechtigten darzulegen und gegebenenfalls zu beweisen sind. ${ }^{6}$

Kindbezogene Gründe sind in diesem Zusammenhang (stets) vorrangig zu prüfen; ein „Altersphasenmodell, das bei der Frage der Verlängerung des Betreuungsunterhalts aus kindbezogenen Gründen allein oder wesentlich auf das Alter des Kindes abstellt, wird diesen Anforderungen nicht gerecht “. ${ }^{7}$ In dem Umfang, in dem das Kind nach Vollendung des dritten Lebensjahres eine kindgerechte Einrichtung besucht oder besuchen könnte, kann sich der betreuende Elternteil nicht mehr auf die Notwendigkeit einer persönlichen Betreuung des Kindes berufen. Dies gilt sowohl für den rein zeitlichen Aspekt der Betreuung als auch für den sachlichen Umfang der Betreuung in einer kindgerechten Einrichtung. ${ }^{8}$

Die Berücksichtigung elternbezogener Gründe ist hingegen Ausdruck der nachehelichen Solidarität, wobei die ausgeübte oder verlangte Erwerbstätigkeit des betreuenden Elternteils neben dem nach der Erziehung und Betreuung in einer Tageseinrichtung verbleibenden Anteil nicht zu einer „überobligatorischen " Belastung des betreuenden Elternteils führen darf. ${ }^{9}$

In der Praxis bedeuten die Entscheidungen des Bundesgerichtshofs, dass kindbezogene Gründe nur im Einzelfall vorliegen dürften, wie z.B. bei einer festgestellten geistigen oder körperlichen Behinderung oder gänzlich fehlender Betreuungsmöglichkeit. Problematischer einzuordnen sind „verhaltensauffällige“ Kinder, wie zum Beispiel solche mit ADHSSymptomen. Hier muss sich der betreuende Elternteil unter Umständen entgegenhalten lassen, dass eine Fremdbetreuung für das Kind sogar „,besser“ wäre.

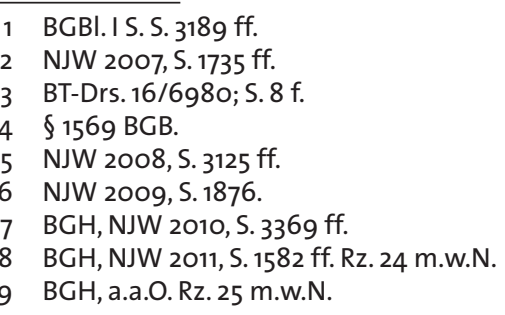

